\title{
Diferentes papéis do óxido nítrico com ênfase nas neoplasias
}

\author{
The different roles of nitric oxide with focus on cancer
}

\author{
Mirela Tinucci Costa ${ }^{1}$ Rita de Cássia Fabeni ${ }^{2}$ Karina Preissing Aptekmann ${ }^{3}$ \\ Rosângela Racarias Machado ${ }^{4}$
}

- REVISÃO BIBLIOGRÁFICA -

\section{RESUMO}

O Óxido Nítrico $(O N)$ é gerado por uma família de isoenzimas, através da catálise enzimática do aminoácido essencial L-arginina, que resulta na formação de L-citrulina e ON. O ON está envolvido em muitos processos fisiológicos dos mamiferos, que incluem a neurotransmissão, controle da pressão sangüinea, inflamação, reações imunológicas e nos mecanismos de defesa contra microorganisnos e tumores. $O$ descontrole na sintese de ON está implicado na patogênese de doenças cardiovasculares, autoimunidade, rejeição de transplantes, doenças degenerativas, na sépsis, na genotoxicidade e no surgimento de neoplasias. O ON também foi incriminado como agente de iniciação da carcinogênese, que, associado a outros fatores, poderia levar ao descontrole da citoestase e da diferenciação celular. A diversidade de efeitos do ON parece estar relacionada às concentrações de ON gerados, à sensibilidade individual das células e à duração do fenômeno.

Palavra-chave: óxido nítrico, câncer.

\section{ABSTRACT}

Nitric Oxide (NO) is generated by a family of isoenzymes, through the enzymatic catalisis of the aminoacid L-arginine, which leads to the production of L-citruline and NO. It is involved in many physiologic processes of mammals, including neurotransmition, blood pressure control, inflammation, immune responses and also in the mechanism of defense against invasive organisms and tumors. The uncontrolled syntesis of $N O$ is thought to take part in the pathogenesis of cardiovascular diseases, auto-immunity, graft rejection, degenerative diseases, as well as in sepsis, genotoxicity and also in neoplasia development. The NO is also considered the initial agent of carcinogenesis, which could lead to uncontrolled cytostasis and cellular differentiation when associated to other factors. The diversity of NO effects seems to be related to the concentration that is generated, to the individual sensibility of cells and the duration of this phenomenon.

Key words: nitric oxide, cancer.

\section{INTRODUÇÃO}

$\mathrm{Na}$ década de 80 , a descoberta do óxido nítrico ( $\mathrm{ON}$ ) como um mensageiro molecular para os vários sistemas do organismo de mamíferos revolucionou as pesquisas acerca da extensão de sua atividade biológica (VANIN, 1998; FLORA FILHO \& ZILBERSTEIN, 2002). Desde então, um crescente número de pesquisas acerca da molécula $\mathrm{ON}$, na fisiopatologia humana e animal, são desenvolvidas (QUEIROZ \& BATISTA, 1998).

O ON participa de muitos processos fisiológicos relacionados aos sistemas nervosos central (CHRISTOPHERSON \& BREDT, 1997) e periférico (CHRISTOPHERSON \& BREDT, 1997; QUEIROZ \& BATISTA, 1998; THOMPSON, 2001;

${ }^{1}$ Professor Assistente, Doutor, Departamento de Clínica e Cirurgia Veterinária, Faculdade de Ciências Agrárias e Veterinárias (FCAV) UNESP, Campus de Jaboticabal. Via de Acesso Prof. Paulo Donato Castelani, s/n, 14884-900, Jaboticabal-SP. E-mail: mirelatc@fcav.unesp.br Autor para correspodência.

${ }^{2}$ Pós-graduando em Medicina Veterinária, FCAV - UNESP, Campus de Jaboticabal.

${ }^{3}$ Graduando em Medicina Veterinária, FCAV - UNESP, Campus de Jaboticabal.

${ }^{4}$ Professor Titular, Departamento de Patologia Veterinária, FCAV - UNESP, Campus de Jaboticabal. 
FLORA FILHO \& ZILBERSTEIN, 2002). O ON tem um importante papel no controle de muitas infecções, apresentando atividade antibacteriana, antiparasitária e antiviral (FLORA FILHO \& ZILBERSTEIN, 2002). Todavia, o descontrole na síntese de $\mathrm{ON}$ está implicado na patogênese de doenças cardiovasculares, autoimunidade, rejeição de transplantes, sépsis (NATHAN, 1992), doenças cerebrais degenerativas (CHRISTOPHERSON \& BREDT, 1997; HALLIWELL, 2001), indução de câncer (SCHMIDT \& WALTER, 1994), genotoxicidade (WINK et al., 1998) e na inflamação (FLORA FILHO \& ZILBERSTEIN, 2002).

O ON também apresenta ação anti-tumoral (JENKINS et al., 1995; MURTA et al., 2002). Entretanto, a atividade anti-tumoral do ON depende da quantidade de $\mathrm{ON}$ gerada e da interação das células do hospedeiro com as do tumor. Esta interação pode gerar efeito ora estimulante ora inibitório na imunidade anti-tumoral (O'SULLIVAN \& LEWIS, 1994; MURTA et al., 2002).

A diversidade de efeitos do ON parece estar relacionada às concentrações de ON gerados, a sensibilidade individual das células e à duração do fenômeno (THOMSEN et al., 1994; QUEIROZ \& BATISTA, 1998).

\section{A SÍNTESE DO ÓXIDO NÍTRICO}

O ON é gerado por uma família de isoenzimas expressas em uma grande variedade de células de mamíferos, através da catálise enzimática do aminoácido essencial L-arginina (MONCADA et al., 1991; SPRINGALL, 1995). A catálise enzimática da reação do aminoácido L-arginina e oxigênio, que resulta na formação de L-citrulina e Óxido Nítrico, é feita por enzimas que são constitutivas (cONS) ou induzidas (iONS). As cONS são enzimas que estão presentes em muitas células; sua liberação depende da concentração intracelular de cálcio/calmodulina e, uma vez liberadas, sintetizam ON por curtos períodos. $\mathrm{O}$ ON formado por esta via participa de muitos processos homeostáticos. Por outro lado, as iONS independem da concentração intracelular de cálcio, e são liberadas por macrófagos poucas horas após sua ativação e também por outras células sob ação de citocinas. As iONS sintetizam ON por longos períodos (MONCADA et al., 1991). Uma terceira isoforma das enzimas que geram ON foi descrita e está expressa em neurônios (ONS1), células endoteliais (ONS3) ou induzida nos macrófagos sob ação de citocinas (ONS2) (KNOWLES \& MONCADA, 1994). A atividade das enzimas, constitutiva e a induzida, pode ser inibida por análogos da L-arginina e a inibição da indução da iONS pode ser abolida por glicocorticóides (MONCADA et al., 1991) e por citocinas, como o TGF- $\beta$, IL-4, IL-10 e o fator de desativação de macrófagos (NATHAN, 1992).

\section{O ÓXIDO NÍTRICO EM CONDIÇÕES FISIOLÓGICAS}

Esta pequena molécula tem importância desde a manutenção inicial da vida, através do controle da circulação placentária e nas contrações uterinas durante o parto, como na neurotransmissão, no controle endócrino, na imuno-regulação e nos vários sistemas do organismo (FLORA FILHO \& ZILBERSTEIN, 2002). No sistema nervoso central, o ON atua como um neuromodulador para o controle da atividade normal, influenciando, inclusive, a formação de memória e intensidade da resposta a um estímulo doloroso (CHRISTOPHERSON \& BREDT, 1997). No sistema nervoso periférico atua como um neurotransmissor (NATHAN \& XIE, 1994). As cONS são transientes e estão envolvidas nos processos homeostáticos, como a neurotransmissão, peristaltismo e na regulação da pressão sangüínea (NATHAN \& XIE, 1994; FLORA FILHO \& ZILBERSTEIN, 2002). O determinante para produção de maiores ou menores quantidades de $\mathrm{ON}$ depende da duração da atividade nas células normais do hospedeiro, em condições fisiológicas. Todavia, em algumas circunstâncias, "in vitro", a ONS expressa nos neurônios pode contribuir para a citotoxicidade celular (NATHAN \& XIE, 1994). Em contraste, a produção aumentada da iONS pode ser detectada em situações fisiológicas, como no útero de coelhas prenhes (NATHAN \& XIE, 1994; WINK et al., 1998). Apresenta também um importante papel na manutenção do fluxo sangüíneo (NATHAN \& XIE, 1994 ;CHRISTOPHERSON \& BREDT, 1997), sendo um importante mediador da ereção peniana (CHRISTOPHERSON \& BREDT, 1997; QUEIROZ \& BATISTA, 1998; THOMPSON, 2001).

No sistema cardiovascular, a ação do ON está associada à hipotensão causada pela administração de lipopolissacaridio bacteriano (LPS) ou citocinas, possivelmente interagindo no choque séptico (NATHAN \& XIE, 1994). Os efeitos do ON de origem vascular estão ligados à inibição plaquetária e à contração do músculo liso. No sistema renal, o ON parece regular a microcirculação. No sistema endócrino, atua mediando a liberação de insulina e parece estar ligado à patogênese do diabetes mellitus (NATHAN, 1992). 
O ON derivado de células epiteliais, neuroendócrinas e endócrinas está envolvido na regulação parácrina e autócrina da neurotransmissão (SCHMIDT \& WALTER, 1994).

\section{O ÓXIDO NÍTRICO EM CONDIÇÕES PATOLÓGICAS}

O descontrole na síntese de ON está implicado na patogênese de doenças cardiovasculares, autoimunidade, rejeição de transplantes, sépsis (NATHAN, 1992) e na genotoxicidade (WINK et al., 1998). Injúria neuronal e doenças cerebrais degenerativas, tais como a doença de Parkinson e Alzheimer no homem, também estão associadas à síntese aumentada de ON (CHRISTOPHERSON \& BREDT, 1997; HALLIWELL, 2001).

Em tumores, o ON assume particular importância na neovascularização, contribuindo para manutenção do fluxo sangüíneo adequado (BUTTERY et al., 1993). A indução da síntese de ON está no contexto das defesas do organismo contra infecções, inflamações e muitas neoplasias, contudo, dependendo das quantidades geradas, pode levar à morte celular e danos teciduais (MONCADA et al., 1991; MONCADA \& HIGGS, 1993 ; NATHAN \& XIE, 1994 \& WINK et al., 1998). A sensibilidade ao ON varia de célula para célula, possivelmente em decorrência da relativa importância das enzimas ferrosulfur (Fe-S) nas diferentes células (MONCADA et al., 1991). O ON produzido pela iONS difunde-se para fora dos macrófagos e invade a célula alvo destruindoa, através da inibição de centros $\mathrm{Fe}-\mathrm{S}$ de enzimas envolvidas na respiração celular (QUEIROZ \& BATISTA, 1998) e inibindo a replicação do DNA (WINK et al., 1998).

O ON também foi incriminado como agente de iniciação da carcinogênese em células com inabilidade de gerar ON. Esta inabilidade, associada a outros fatores, poderia levar ao descontrole da citoestase e da diferenciação celular, favorecendo a propagação de um clone maligno e culminar com o surgimento de tumores (WINK et al. 1998).

\section{O ÓXIDO NÍTRICO NO SISTEMA IMUNE E NA INFLAMAÇ̃̃̃O}

No sistema imune e na inflamação, o ON endógeno parece exercer efeitos pró e antiinflamatórios (NATHAN, 1992). Os efeitos próinflamatórios do ON não são evidentes sob condições fisiológicas agudas, podendo, nestes casos, mediar funções anti-inflamatórias, como a inibição de adesão de neutrófilos, atividade da cicloxigenase, formação de citocinas e reabsorção óssea (SCHMIDT \& WALTER, 1994).

Durante a resposta imune mediada por células, a maioria das células adquire a capacidade de expressar a forma de ON induzida (NATHAN \& XIE, 1994). A ação do ON depende da célula $T$ reconhecer um antígeno específico, embora sua ação na resposta imune mediada por células não seja específica (SCHMIDT \& WALTER, 1994). A expressão dos antígenos Ia por macrofagos murinos torna-se crucial para que haja a interação com linfócitos T e conseqüente secreção de IFN- $\gamma$, um importante co-indutor da síntese de ON (XIE et al., 1992; THOMPSON et al., 1998). Todavia, pesquisadores relatam que o LPS inibe a expressão de antígenos Ia por macrófagos estimulados por IFN- $\gamma$ (FIGUEIREDO et al., 1989). Entretanto, SICHER et al. (1994) atribuíram ao ON a responsabilidade por este efeito do LPS, propondo ainda que o ON sirva como um mecanismo de "feedback", prevenindo a excessiva ativação das células $\mathrm{T}$ e produção de $\mathrm{ON}$. Neste contexto, possivelmente o ON possa ser visto como um modulador imune (SCHMIDT \& WALTER, 1994). Ficou evidenciado, por CUNHA et al. (1993), que a fagocitose por si só não induz a síntese de ON. A fagocitose de partículas inertes por macrófagos peritoniais, em cultura, ou por macrófagos murinos, estimulados por IFN- $\gamma$, não são estímulo suficiente para a indução expressiva de iONS. No entanto, a fagocitose de agentes biológicos, nas mesmas condições, induz altos níveis de ON, sugerindo que a produção de $\mathrm{ON}$ seja dependente de estímulo imunológico e que faça parte dos mecanismos de defesa do organismo.

IFN- $\gamma$, TNF- $\alpha$ e MIF (fator inibidor de macrófagos), após interagirem com seus receptores nos macrófagos, desencadeiam sinais intracelulares que culminam com a ativação da enzima ON. Por outro lado, citocinas como a IL-4, IL-10 e TGF- $\beta$, quando ocupam seus receptores, desencadeiam sinais intracelulares que resultam na inibição da síntese de ON (CUNHA, 1995).

Entre os efeitos protetores do ON estão a destruição de muitos agentes patógenos e de células tumorais (NATHAN \& HIBBS, 1991; NATHAN, 1992). POLTE et al. (1997) demonstraram que o ON também exerce efeito citoprotetor, mantendo a integridade da parede vascular, indicativo do potencial efeito anti-inflamatório e anti-aterogênico. Efeito antitrombogênico do $\mathrm{ON}$ em endotélio não lesionado foi 
observado por FOURNET-BOURGUIGNON et al. (2002).

\section{O ÓXIDO NÍTRICO E A APOPTOSE}

Aparentemente, a morte celular mediada por ON ocorre tanto por necrose como por apoptose (ALBINA et al., 1989; KRÖNCKE et al., 1997). Em miócitos cardíacos a apoptose induzida pelo $\mathrm{ON}$ parece ser dependente de cGMP, enquanto que a necrose não (TAIMOR et al., 2000). O ON foi capaz de induzir apoptose, "in vitro", em várias linhagens de células (ALBINA et al., 1989). Contudo, a apoptose em linhagens celulares transformadas de rápido crescimento parece não estar ligada ao mecanismo mediado pelo ON (ALBINA et al., 1989; KRÖNCKE et al., 1997).

Na regulação da apoptose, o ON parece apresentar efeito contraditório. Os efeitos próapoptóticos parecem estar mais relacionados a condições patofisiológicas, onde grandes quantidades de ON são produzidas por síntese induzida de $\mathrm{ON}$. Em contraste, a liberação contínua de ON pelo endotélio inibe a apoptose e pode contribuir para a função anti-aterosclerótica do ON (DIMMELER \& ZEIHER, 1997). Células de mastocitomas, sarcomas, melanomas e a L929 (linhagem celular de tecido conjuntivo de camundongo) sofrem exuberante apoptose quando expostas ao ON, contudo outras linhagens celulares, como A549 (linhagem celular humana de carcinoma de pulmão), exibem limitadas porcentagens de apoptose ( 5 a 20\%), quando expostas aos doadores químicos de ON (WINK et al., 1998), evidenciando o efeito contraditório do ON na regulação da apoptose (DIMMELER\& ZEIHER, 1997).

O ON pode afetar a expressão e a atividade de proteínas críticas no ciclo celular e, por sua vez, também ser alvo das mutações do DNA celular. A exposição das células aos doadores de $\mathrm{ON}$ pode resultar em supra-regulação do gene supressor de tumor, o p53, possivelmente em resposta ao dano do DNA mediado pelo ON (WINK et al., 1998).

\section{O ÓXIDO NÍTRICO EM TUMORES}

O desenvolvimento do tumor, sua vascularização e seu potencial invasivo estão relacionados a reduzidas concentrações de $\mathrm{ON}$ produzidas pelas células tumorais (JENKINS et al., 1995). Em lesões pré-malignas e em tumores de intestino grosso, a síntese de ON está reduzida ou ausente, ao passo que altos níveis de ON foram encontrados em tumores ginecológicos (CHHATWAL et al., 1996). Correlação inversa entre o potencial metastático de tumores e a produção do $\mathrm{ON}$ foi observada por muitos pesquisadores (JENKINS et al., 1995; HAJRI et al., 1998).

A co-cultura de células endoteliais pulmonares de camundongos, ativadas pelo IFN- $\gamma$ e pelo TNF- $\alpha$, com células de uma linhagem de sarcoma de células reticulares murino, resulta em significante lise das células tumorais. A adição de um inibidor de ON (NMA) inibe completamente a lise das células tumorais e a síntese de $\mathrm{ON}$ em $48 \%$, sugerindo que o ON produzido pelas células endoteliais de pulmão, estimuladas pelas citocinas, seria responsável pela lise das células tumorais (LI et al., 1991). Foi hipotetizado, por WANG et al. (2000), que a microvasculatura hepática regula a apreensão e o destino de células metastáticas de câncer através da liberação de ON. Estes autores concluíram que existe um mecanismo de defesa natural contra metástases de câncer por meio do qual a apreensão de células tumorais no fígado induz a liberação de $\mathrm{ON}$ endógeno, levando à morte das células tumorais no sinusóide e reduzindo a formação de metástase hepática. Foi demonstrado que o ON participa também no mecanismo de resistência não específica, mediada pelo $\mathrm{BCG}$, ao desenvolvimento do teratocarcinoma ovariano murino singenéico, bem como na resistência ao implante de tumor ovariano xenogenéico, "in vivo" (FARIASEISNER et al., 1994).

Pesquisas recentes desenvolvidas em nosso laboratório evidenciaram que células do tumor venéreo transmissível canino (TVT) sintetizam iONS "in vivo" e "in vitro" e sugerem que o ON sintetizado esteja envolvido na regressão do TVT (TINUCCI-COSTA, 1999).

ALLEVA et al. (1994) observaram que macrófagos de camundongos portadores de fibrossarcoma apresentavam aumento de síntese de ON e esta síntese aumentada tinha a capacidade de suprimir o estímulo de proliferação de células T CD4 ${ }^{+}$. Efeitos adversos do ON "in vivo" também foram observados por EDWARDS et al. (1996), em uma linhagem de células de tumor mamário murino (EMT6). O tratamento destas células com LPS/IFN- $\gamma$ estimulou a produção de $\mathrm{ON}$ e inibiu o crescimento das células em $50 \%$. Paralelamente, a inoculação dessas culturas de células (EMT-6) em camundongos resultou no aparecimento de tumores subcutâneos e metástases pulmonares duas vezes maiores que nos controles. Na presença de um inibidor da ONS, o crescimento das células, o tamanho do tumor e o número de metástases não diferiram dos controles. 
THOMSEN et al. (1995) encontraram maior atividade da iONS em tumores mamários humanos de maior malignidade. Do mesmo modo, SOINI et al. (2000) demonstraram que a síntese de $\mathrm{ON}$ atua no crescimento e progressão do mesotelioma maligno e adenocarcinoma metastático de pleura.

Em alguns tumores humanos, como no esôfago de Barrett, foram identificados elevados níveis de mRNA para iONS e para ciclooxygenase-2 (COX2). Para os pesquisadores, estes resultados sugerem que tanto a iONS como a COX-2 estejam envolvidas na progressão neoplásica associada ao esôfago de Barrett (WILSON et al., 1998).

A adesão entre as células endoteliais de veias umbilicais humanas com células da linhagem celular de fibrossarcoma humano, em co-cultura, parece induzir a produção de ON. O ON produzido age aumentando a adesão entre as duas linhagens celulares e também a permeabilidade endotelial. Este efeito favorece a invasão celular através do endotélio e, conseqüentemente, o potencial metastático (YUDOH et al., 1997). Neste mesmo contexto, MAEDA \& AKAIKE (1998) sugerem que o ON gerado em tumores sólidos experimentais, poderia facilitar a permeabilidade vascular e conseqüentemente o suprimento nutricional do tecido e o rápido crescimento do tumor. Resultados semelhantes foram observados em camundongos com síntese deficiênte de iONS inoculados com linhagens de tumores por TAMURA et al. (2002).

DiNAPOLI et al. (1996) observaram que populações de macrófagos peritoniais de murinos normais (N-PEM), de portadores de tumor mamário (TB-PEM) e de macrófagos infiltrados no tumor (TAM), estimulados ou não, expressaram diferentes quantidades de $\mathrm{ON}$, sendo a supressão mais pronunciada na TAM. Para os autores, a reduzida capacidade tumoricida dos macrófagos de camundongos com tumor mamário deve-se à diminuição da expressão da iONS, possivelmente decorrente de fatores produzidos ou induzidos pelas células do próprio tumor, de outras células presentes no tumor, ou do estado de maturação desses macrófagos. Estes fatores associados poderiam contribuir para o desenvolvimento do tumor e eventual aniquilação do hospedeiro. Neste contexto, foi sugerido que a supressão da expressão da iONS em macrófagos de camundongos portadores de tumor pode decorrer da formação sistêmica de agentes supressores derivados do tumor, como a IL-10, TGF$\beta 1$ e PGE2 (ALLEVA et al., 1994; WINK et al., 1998).

KLOTZ et al. (1998) detectaram a iONS em todos os espécimes de carcinoma de próstata humano testados, inclusive nas células epiteliais malignas do tumor. Nenhuma marcação foi identificada nos casos de pacientes com hiperplasia prostática benigna, sugerindo que o $\mathrm{ON}$ sintetizado assuma importante papel no desenvolvimento da doença. Resultados semelhantes foram observados por AALTOMAA et al. (2000).

A detecção da síntese de $\mathrm{ON}$ em tumores ginecológicos pobremente diferenciados poderia ser empregada, no futuro, como um fator prognóstico, visto que altos níveis de NOS, não correlacionados à presença de macrófagos foram detectados nas células de neoplasias ginecológicas pobremente diferenciadas, em comparação a tecidos normais e tecidos ginecológicos não neoplásicos (THOMSEN et al., 1994).

A partir da inoculação de células de uma linhagem de adenocarcinoma de cólon em ratos LEJEUNE et al. (1994), observaram que a imunossupressão dos linfócitos estava associada ao aumento na produção de ON por macrófagos esplênicos. Adicionalmente, cultura das células mononucleares esplênicas dos ratos portadores do tumor, quando tratada com um inibidor da síntese de ON fazia restaurar a capacidade proliferativa dos linfócitos. Os mesmos pesquisadores observaram também que durante a fase de crescimento do tumor, a expressão de RNAm para iNOS e a produção de ON estavam aumentadas. Este foi o primeiro trabalho que descreve o efeito do ON na imunossupressão induzida por tumores. Outros autores sugerem que a produção de ON pelas células de tumores poderia ser um dos mecanismos utilizados pelo tumor para se evadir das defesas do organismo (LEJEUNE et al., 1994; CHHATWAL et al., 1996; EDWARDS et al., 1996; DiNAPOLI et al., 1996; WINK et al., 1998).

CHHATWAL et al. (1996) hipotetizaram dois possíveis mecanismos que poderiam estar relacionados ao surgimento de uma neoplasia no epitélio gastrointestinal, através da produção aberrante de $\mathrm{ON}$. O primeiro deles estaria relacionado à inabilidade da célula gerar o $\mathrm{ON}$, em conseqüência de fatores genéticos e ou ambientais, resultando em uma seqüência de eventos que poderiam culminar com o surgimento de neoplasia. Nesta etapa, o ON funcionaria como um agente de iniciação da carcinogênese, o segundo mecanismo estaria relacionado às conseqüências da deficiência de $\mathrm{ON}$ na célula, que levaria ao descontrole da citostase e da diferenciação e à propagação de um clone maligno. A deficiência de $\mathrm{ON}$ em tumores pode auxiliar a célula tumoral a evadir-se da ação das células do sistema imune e simultaneamente escapar da morte 
programada, retendo, porém, a habilidade de multiplicação. O papel do ON como agente promotor de câncer possivelmente se deva à geração de espécies reativas de nitrogênio óxido (RNOS), geradas a partir das sintases de ON. As reações seguintes à formação das RNOS dão origem ao estresse oxidativo e nitrosativo e apresentam efeitos potencialmente genotóxicos (WINK et al., 1998).

$\mathrm{O} \mathrm{ON}$ em tumores apresenta efeitos controversos. Aparentemente, o que determina a progressão ou a regressão tumoral associada ao $\mathrm{ON}$ seria o metabolismo da arginina pelos macrófagos intra-tumorais (MURTA et al., 2002).

\section{CONCLUSÃO}

O determinante para a produção de maiores ou menores quantidades de ON depende da duração da atividade das células normais do hospedeiro, em condições fisiológicas. Em quantidades reduzidas, está envolvido nos processos homeostáticos, como a neurotransmissão, peristaltismo e na regulação da pressão sangüínea momento-a-momento. Sua produção aumentada pode ser detectada em algumas situações fisiológicas, como a prenhez e defesa do organismo contra infecções e inflamações. O descontrole da síntese de ON está implicado na patogênese de diversos estados mórbidos, tais como doenças cardiovasculares e rejeição de órgãos transplantados. Em tumores, o ON apresenta uma complexidade de efeitos, ora contribuindo para a regressão do tumor ora para a progressão. Experimentos "in vivo" e "in vitro" empregando uma mesma linhagem de células transformadas podem apresentar efeitos opostos. Se por um lado foi observada correlação inversa entre o potencial metastático do tumor e a produção de $\mathrm{ON}$, por outro, a maior produção de ON foi detectada em tumores de maior potencial de malignidade. Contudo, esta diversidade de efeitos do $\mathrm{ON}$ em tumores parece estar relacionada às concentrações de $\mathrm{ON}$ gerados, à sensibilidade individual das células e à duração do fenômeno. Em suma, os estudos sobre os efeitos do ON em tumores devem ser continuados, buscando-se elementos que auxiliem no entendimento dos efeitos paradoxais do ON.

\section{REFERÊNCIAS BIBLIOGRÁFICAS}

AALTOMAA, S.H. et al. The prognostic value of inducible nitric oxide synthase in local prostate cancer. British Journal of Urology International, v.86, p.234-239, 2000.
ALBINA, J.E. et al. Regulation of macrophage functions by Larginine. Journal Experimental Medicine, v.169, p.1021-1029, 1989.

ALLEVA, D.G.; BURGER, C.J.; ELGERT, K.D. Tumor-induced regulation of supressor macrophage nitric oxide and TNF-a production. Role of tumor-derived IL-10, TGF-b, prostaglandin $\mathrm{E}_{2}$. Journal Immunology., v.153, p.1674-1686, 1994.

BUTTERY, L.D.K. et al. Induction of nitric oxide synthase in the neo-vasculature of experimental tumors in mice. Journal Pathology, v.171, p.311-19, 1993.

CHHATWAL, V.J.S. et al. Nitric oxide and cancer. Medical Hypothesis, v.46, p.21-24, 1996.

CHRISTOPHERSON, K.S.; BREDT, D. Nitric oxide in excitable tissues: physiological roles and disease. Journal Clinical Investigation, v.100, n.10, p.2424-2429, 1997.

CUNHA, F.Q. et al. Phagocytosis and induction of nitric oxide synthase in murine macrophages. Immunology, v.79, p.408-411, 1993.

CUNHA, F.Q. Mecanismos de indução e ou inibição no sistema óxido nítrico. 1995. 79f. Tese (livre docência) - Faculdade de Medicina de Ribeirão Preto, Universidade de São Paulo.

DIMMELER, S.; ZEIHER, A.M. Nitric oxide and apoptosis: another paradigm for the double-edged role of nitric oxide. Nitric Oxide, v.1, n.4, p.275-281, 1997.

DiNAPOLI, M.R.; CALDERON, C.L.; LOPEZ, D,M. The altered tumoricidal capacity of macrophages isolated from tumor-bearing mice is related to reduce expression of the inducible nitric oxide synthase gene. Journal Experimental Medicine, v.183, p.1323$1329,1996$.

EDWARDS, P. et al. Tumor cell nitric oxide inhibits cell growth in vitro, but stimulates tumorigenesis and lung metastasis in vivo. Journal Surgery Research, v.63, n.1, p.49-52, 1996.

FARIAS-EISNER, R. et al. Nitric oxide is an important mediator for tumoricidal activity in vivo. Proceedings of the National Academy Sciences, v.91, p.9407-9411, 1994.

FIGUEIREDO, F.; KOERNER, T.J.; ADAMS, D.O. Molecular mechanisms regulating the expression of class II histocompatibility molecules on macrophages: effects of inductive and supressive signals on gene transcription. Journal Immunology, v.143, n.11, p.3781-3786, 1989.

FLORA FILHO, R.; ZILBERSTEIN, B. Óxido nítrico: o simples mensageiro percorendo a complexidade. Metabolismo, síntese e funções. Revista Associação Médica Brasileira. Capturado em 13 jul. 2002. Online. Disponível na Internet: http://w.../ scielo.php? $\quad$ script $=$ sci_arttext\&pid $=$ SO $104-$ $42302000000300012 \& 1 \mathrm{ng}=\mathrm{pt} \& \mathrm{nrm}=\mathrm{is}$.

FOURNET-BOURGUIGNON, M et al. Implication of the NO biodisponibility in the antithrombogenic properties of the endothelium of non injured rabbit carotid arteries using an ex vivo model of platelet deposition. Capturado em 10 jul. 2002. Online. Disponível na Internet: http:// www.academicpress.com/www/journal/no2002/ 15814.html. 
HAJRI, A. et al. Role of nitric oxide in pancreatic tumor growth: in vivo and in vitro studies. British Journal Cancer, v.78, n.7, p.841849,1998

JENKINS, D.C.; CHARLES, I.G.; THOMSEN, I.I. Roles of nitric oxide in tumor growth. Proceedings of the National Academy Sciences, v.92, p.4392-4396, 1995.

HALLIWELL, B. Role of free radicals in the neurodegenerative diseases: Therapeutic implications for antioxidant treatment. Drugs \& Aging, v.18, n.9, p.685-716, 2001

KLOTZ, T. et al. Selective expression of inducible nitric oxide synthase in human prostate carcinoma. Cancer, v.82, n.10, $1897-$ 1903, 1998 .

KNOWLES, R.G.; MONCADA, S. Nitric oxide synthesis in mammals. Biochemistry Journal, v.298, p.249-258, 1994.

KRÖNCHE, K.D; FEHSEL, K.; KOLB-BACHOFEN, V. Nitric oxide: cytotoxicity versus cytoprotection - how, why, when, and where? Nitric Oxide, v.1, n.2, p.107-20, 1997.

LEJEUNE, P. et al. Nitric oxide involvement in tumor-induced immunosuppression. Journal Immunology., v.152, n.10, p.50775083, 1994.

LI, L. et al. Role of nitric oxide in lysis of tumor cells by cytokinesactivated endothelial cells. Cancer Reseach., v.51, n.15, p.2531$2535,1991$.

MAEDA, H.; AKAIKE, T. Nitric oxide and oxygen radicals in infection, inflammation, and cancer. Biochemistry (Mosc), v.63, n.7, p.854-865, 1998. /Resumo/.

MONCADA, S.; PALMER, R.M.J.; HIGGS, E.A. Nitric oxide: Physiology, pathophysiology, and pharmacology. Pharmacological Review, v.43, n.2, p.109-142, 1991.

MONCADA, S.; HIGGS, E.A. The L-Arginine-nitric oxide pathway. New England Journal Medicine., v.27, p.2002-2012, 1993.

MURTA, B.M.T. et al. The relationship of host immune cells, cytokine and nitric oxide production to tumor cells in ovarian carcinoma. São Paulo Medical J. Capturado em 13 jul. 2002. Online. Disponível na Internet http://w... scielo.php?script $=$ sciarttext\&pid=S1516-31801999 000200008 $\& 1 \mathrm{ng}=\mathrm{pt} \& \mathrm{nrm}=\mathrm{is}$

NATHAN, C.; HIBBS, J.B. Role of nitric oxide synthesis in macrophage antimicrobial activity. Current Opinion Immunology, v.3, p.65-70, 1991

NATHAN, C. Nitric oxide as a secretory product of mammalian cells. FASEB Journal, v.6, p.3051-3064, 1992.

NATHAN, N.C.; XIE, Q. Nitric oxide synthases: roles, tolls, and controls. Cell, v.78, p.915-918, 1994.

O'SULLIVAN, C.; LEWIS, C.E. Tumor-associated leucocytes: Friends or foes in breast cancer carcinoma. Journal Pathology, v.172, p.229-235, 1994.

POLTE, T.; OBERLE, S.; SCHRÖDER, H. Nitric oxide protects endothelial cells from tumor necrosis factor- $\alpha$-mediated cytotoxicity: possible involvement of cyclic GMP. FEBS Letters v.409, p.46-48, 1997.
QUEIROZ, S.L.; BATISTA, A.A. Funções biológicas do óxido nítrico. Quimica Nova. São Paulo. Capturado em 13 jul. 2002. Online. Disponível na Internet: http://w.../scielo. p h p ? s c r i p t $=$ s c i a r t e x t \& p i d = S 01000 $40421999000400017 \& 1 \mathrm{ng}=\mathrm{pt} \& \mathrm{nrm}=\mathrm{is}$

SCHMIDT, H.H.H.W.; WALTER, U. NO at work. Cell, v.78, p.919925, 1994.

SICHER, S.C.; VAZQUEZ, M.A.; LU, C.Y. Inhibition of macrophages Ia expression by nitric oxide. Journal Immunology, v.153, p.1293-1300, 1994.

SOINI, Y. et al. Expression of inducible nitric oxide synthase in healthy pleura and in malignant mesothelioma. British Journal of Cancer, v.83, n.7, p.880-886, 2000.

SPRINGALL, D.R. Nitric oxide - Friend and foe. editorial. Journal Pathology, v.175, p.165-166, 1995.

TAIMOR, G.; HOFSTAETTER, B.; PIPER, H.M. Apoptosis induction by nitric oxide in adult cardiomyocytes via cGMPsignaling and its impairment after simulated ischemia. Cardiovascular Research, v.45. p.588-594. 2000.

TAMURA, F. et al. Tumorigenesis and metastasis-promoting effects of nitric oxide. Capturado em 16 jul, 2002. Online. Disponível na Internet: http://www.apnet.com/www/journal/no2002/ 15721.html.

THOMPSON, D.C. et al. Cytokine-induced nitric oxide formation in normal but not in neoplastic murine lung epithelial cell lines. American Journal Physiology., v.274, p.L922-L932, 1998.

THOMPSON, T.A. O oxido nítrico e suas funções no corpo humano. Capturado em 10 jul, 2002. Online. Disponível na Internet: http://www.neurociencias.nu/LifeScience/ oxidonitrico.htm.

THOMSEN, L.L. et al. Nitric oxide synthase activity in human gynecological cancer. Cancer Research., v.54, n.1, p.1352-1354, 1994

THOMSEN, L.L. et al. Nitric oxide synthase activity in human breast cancer. British Journal Cancer, v.72, p.41-44, 1995.

TINUCCI-COSTA, M. Tumor venéreo transmissível canino: estudos imuno-histoquímicos e de transplantações xenogênicas e alogênicas. 1999. 149f. Tese (Doutorado em Patologia - subárea Patologia Experimental) - Faculdade de Medicina de Ribeirão Preto, Universidade de São Paulo.

VANIN, A.F. Biological role of nitric oxide: History, modern state, and pespectives for research. Biochemistry, (Mosc.), v.63, n.7, p.731-33, 1998 .

WILSON, K.T. et al. Increased expression of inducible nitric oxide synthase and ciclooxygenase- 2 in Barret's esophagus and associated adenocarcinomas. Cancer Research., v.58, n.14, p.2929-2934, 1998 .

WANG, H.H. et al. B16 melanoma cell arrest in the mouse liver induces nitric oxide release and sinusoidal cytotoxicity: a natural hepatic defense against metastasis. Cancer Research, v.60, n.15, p.5862-5869, 2000 .

WINK, D.A. et al. The multifaceted roles of nitric oxide in cancer. Carcinogenesis, v.19, n.5, p.711-721, 1998. 
YUDOH, K.; MATSUI, H.; TSUJI, H. Nitric oxide induced by tumor cells activates tumor cell adhesion to endothelial cells and permeability of the endothelium in vitro. Clinical Experimental Metastasis, v.15, n.6, p.557-567, 1997.
XIE, Q.W. et al. Cloning and characterization of inducible nitric oxide synthase from mouse macrophages. Science, v.256, p.225-226, 1992 .

Ciência Rural, v. 33, n. 5, set-out, 2003. 